\title{
Tool Trajectory Optimization of Spray Painting Robot for Many- Times Spray Painting
}

\author{
Yong Zeng ${ }^{1}$, Jun Gong ${ }^{3}$, Ning $\mathrm{Xu}^{2 *}$ and Nailing $\mathrm{Wu}^{1}$ \\ ${ }^{1}$ School of Mechanical Engineering, Yancheng Institute of Technology, Yancheng, \\ 224051, China \\ ${ }^{2}$ Key Laboratory for Advanced Technology in Environmental Protection of Jiangsu \\ Province, Yancheng Institute of Technology, Yancheng, 224051, China \\ ${ }^{3}$ School of Mechanical and Electronical Engineering, Lanzhou University of \\ Technology, Lanzhou, 730050, China \\ xuning196402@163.com
}

\begin{abstract}
Spray gun trajectory optimization of spray painting robot is highly desirable for today's automotive manufacturing. Sometimes, the way of many-times spray painting is used to meet the technological requirements. In this paper, a new spray gun trajectory optimization method of many-times spray painting for the plane has been developed after complex surface partitioned. Based on the spray gun trajectory optimization of one-time spray painting, the method utilizes the coating thickness superposition cycle and status number to establish the coating thickness superposition model for many-times spray painting and to optimize the spray gun trajectory of many-times spray painting. The number and cycle of coating thickness superposition status are developed to modeling the coating thickness distribution model for many-times spray painting. The coating thickness deviation from the required coating thickness is optimized by modifying the offset distance between the subsequent spray gun trajectory and the first spray gun trajectory. The results of simulations have shown that the spray gun trajectory optimization of many-times spray painting achieves satisfactory performance, the coating thickness uniformity satisfy high standard requirement.
\end{abstract}

Keywords: spray painting robot, many-times spray painting, trajectory optimization, thickness uniformity, simulation

\section{Introduction}

Path planning for the industrial robot plays an important role in the intelligent control of robot, such as welding robot [1] and spray painting robot. The trajectory optimization of spray painting robot for complex free surface is a research focus all long, mainly due to automobiles, airplanes and shipping requirements of high-uniformity in spraying process. The uniformity of paint thickness on a product can strongly influence the quality of the product. Spray gun trajectory optimization is crucial for achieving the uniformity of paint thickness and has been an active research area for many years in automatic trajectory generation method.

Conner et al., [2, 3, 4] developed an automatic trajectory planning method for simple automotive surfaces. Their method is based on Gauss Bonnet Theorem generate the seed curve and its offset curves on a simple surface patch; these generated curves are used as paint gun trajectory. The spray gun velocity and spacing distance of each trajectory were 
optimized, so that coating uniformity to achieve the best on a surface. However, their method cannot suitable for paint gun trajectory optimization of large complex free-form surfaces; they did not report how to partition the large complex free-form surface. Chen et al., [5, 6, 7] developed an automatic trajectory generation system for large free-form surfaces. Their method utilizes the CAD information of a free-form surface and a paint gun model to generate a paint gun trajectory to satisfy the paint thickness requirements. At the same time, they developed a topology partition method for large complex curved surface and developed planning pattern and trend of painting gun path for different form patch. But they did not regard the optimization of spray cycle time for whole surface. Zhao et al., [8, 9, 10] developed a paint gun trajectory optimization in consideration of spray cycle time. Their method utilizes the genetic algorithm and ant colony algorithm to solve multi-target paint gun trajectory optimization and achieve optimal thickness uniformity and spray cycle time objective. Li et al., [11] developed a point cloud slicing technology to generate paint gun trajectory for unknown CAD information of surface. At the same time, they optimized the paint gun velocity and spacing distance of each trajectory to make the coating uniformity to achieve requirement.

Although paint gun trajectory optimization of spray painting robot for complex freeform surface has came a long way, but their study are confined to the one time spray. If many-times spray is required in spray technology, such as the automotive surface needs three kinds of coating that include ground coat paint, finish paint and varnish, so that the spray time of automotive painting needs three times at least. Sometimes, in order to meet spray technology requirements, the same kind coating is generated by two to three times spray. If the optimized paint gun trajectory of each time spray is superposed simply, the coating thickness uniformity can not achieve promotion and it can not satisfy high standard requirement. In this paper, a new paint gun trajectory optimization of many-times spray is developed such that the coating thickness uniformity is promoted. Based on the paint gun trajectory optimization of one time spray, the number and cycle of coating superposition status are developed. Optimization processes are developed to optimize the paint thickness for multiple flat patches. By modifying the offset distance between each time spray trajectory, the paint thickness deviation from the required paint thickness is optimized. The constant two times spray, the variable two times spray and the constant three times spray were used to test the optimization scheme. Simulations were performed to verify the coating thickness uniformity.

\section{Spray Gun Trajectory Optimization for one Time Spray Painting}

\subsection{The Paint Gun Model}

Different pain gun models have been used in spray painting. Some models are simple and some models are quite complex. In this paper, a typical paint gun model is used, as shown in Figure 1 (a), where $\varphi$ is the fan angle, $H$ is the distance of paint gun to the spray direction, $Q$ is the paint gun flow rate, $\alpha$ is the paint gun dip angle (here $\alpha=0^{\circ}$ ) and $R$ is the spray radius. $r$ is the distance of a point $S$ on a surface to the spray direction. Do spray painting experiment on a flat surface to obtain the paint deposition rate. The profile of paint deposition rate can be roughly approximated by parabolic curves [12], as shown in Figure 1 (b). Without regard to the parameters impact on the paint deposition rate, such as the gun standoff, the flow rate of paint, the atomizing pressure and solvent concentration. In this paper, these parameters are fixed [13]. Therefore, the paint deposition rate is only related to the distance $r$, the deposition rate can be expressed as a function. 


$$
f(r)= \begin{cases}\mathrm{A}\left(R^{2}-r^{2}\right) & |r| \leq R \\ 0 & |r|>R\end{cases}
$$

Where $\mathrm{A}$ is constant.

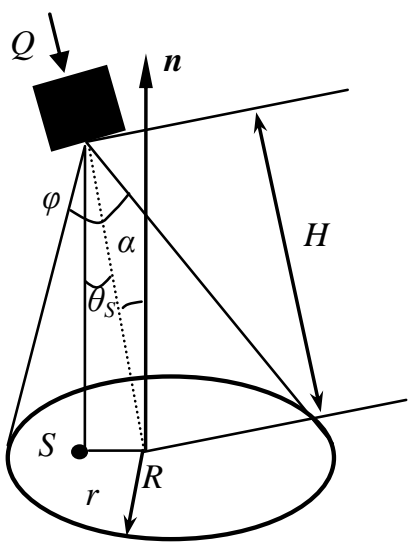

(a) paint gun model

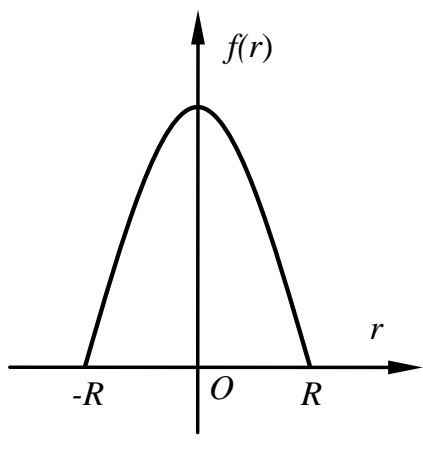

(b) paint deposition rate

Figure 1. Tool-model and Material Deposition Rate

\subsection{Surface Partition and Spray Gun Trajectory Generation}

The complex free-form surface has some characteristics, such as complex connected region and large curvature of some points. In order to better improve coating thickness uniformity for the surface. Before the paint gun trajectory is optimized, generally, a surface can be partitioned into several patches which are simply connected region and can be approximated as a plane [5]. The paint gun trajectory of each patch was generated by the bounding-box method, and then the paint gun trajectories of each patch were connected to a paint gun trajectory of complex free-form surface.

\subsection{Parameters Optimization of Spray Gun Trajectory}

The desired coating of surface is generated by superposition between two adjacent paint paths, as shown in Figure 2. The parameters which are the spray gun velocity and the spacing distance of adjacent paths affect the coating thickness uniformity; therefore these parameters need to be optimized in spray painting process. The coating thickness on any paint gun path can be expressed as: 


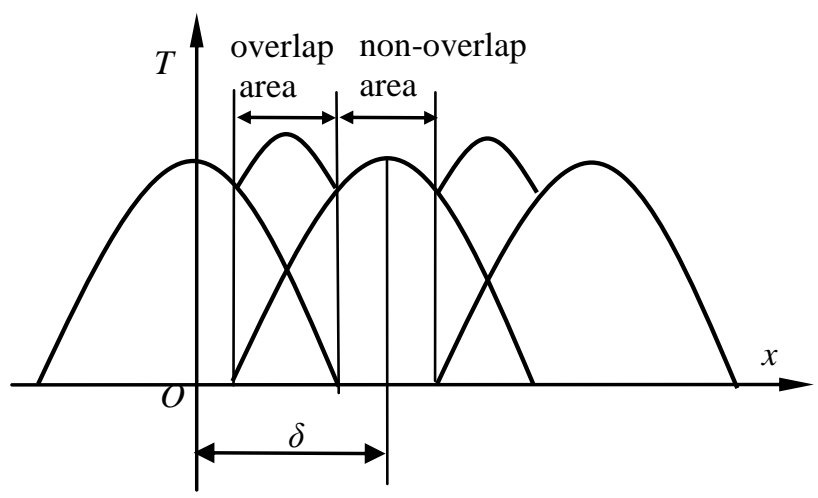

Figure 2. Coating Thickness Superposition of one Time Spray Painting

$$
\begin{aligned}
& T_{i}{ }^{\prime}(x)=2 \int_{0}^{\sqrt{R^{2} \cdot(x-(i-1) \delta)^{2}} / v} f(r) d t \\
& =2 \int_{0}^{\sqrt{R^{2} \cdot(x-(i-1) \delta)^{2}} / v} A\left[R^{2}-\left(\sqrt{(v t)^{2}+(x-(i-1) \delta)^{2}}\right)^{2}\right] d t=\frac{4 A}{3 v}\left[R^{2}-(x-(i-1) \delta)^{2}\right]^{1.5} \\
& (i-1) \delta-R \leq x \leq R+(i-1) \delta
\end{aligned}
$$

After one time spray painting, the superimposed coating thickness of a point $S$ can be expressed as:

$$
T_{S}^{1}(x)= \begin{cases}T_{i}^{1}(x) & (i-1) \delta \leq x \leq i \delta-R \\ T_{i}^{1}(x)+T_{i+1}^{1}(x) & i \delta-R \leq x \leq(i-1) \delta+R \\ T_{i+1}^{1}(x) & (i-1) \delta+R \leq x \leq i \delta\end{cases}
$$

Where $x$ is the distance of the point to the first path; $T_{i}^{1}(x)$ and $T_{i+1}^{1}(x)$ are the coating thickness due to the $i$ and the $i+1$ path.

In order to achieve the best of coating thickness uniformity, the optimization objective function can be expressed as:

$$
\left\{\begin{array}{l}
\min E_{1}(v, \delta)=\int_{(i-1) \delta}^{i \delta}\left(T_{S}^{1}(x, \delta, v)-T_{d}\right)^{2} d x \\
\min E_{\delta \in[R, 2 R]}(v, \delta)=\left(T_{\max }-T_{d}\right)^{2}+\left(T_{\min }-T_{d}\right)^{2}
\end{array}\right.
$$

Where $\delta$ is the spacing distance of adjacent paths; $v$ is the paint gun velocity; $T_{\max }$ and $T_{\min }$ are the maximum and the minimum coating thicknesses; $T_{\mathrm{d}}$ is the desired coating thickness.

If the partial derivative of the above formula was operated with respect to $v, v$ can be represented a function of $\delta$. Therefore, above formula can be transformed to a single variable optimization problem. The golden section method was used to solve the optimal value $\delta$.

\section{Spray Gun Trajectory Optimization for Many-Times Spray Painting}

In the actual process of spray painting, the surfaces of work piece often are covered with the different functional coating. Each the same kind coating is generated by two to three times spray in spray technology and sometimes the thickness uniformity requirement is higher, one 
time spray can not meet these requirements. In order to obtain the best coating thickness uniformity for many-times spray, the coating thickness superposition model after multi-pass spray painting must be developed.

When many-times spray painting, the coating thickness superposition model is influenced of the number $M$ and cycle $W$ of superposition status. The reason is that the coating is composed of overlap and non-overlap region when one time spray painting, while the overlap and non-overlap regions of each time spray painting are superimposed together too when many-times spray painting, so the superposition status of coating thickness is due to the spray painting time. In order to modeling the coating thickness superposition model, the $M$ and the $W$ must be considered. The cycle $W$ is the range of coating superposition status, this status can show the coating thickness distribution of any place on surface, the $M$ is the number of coating thickness superposition status in a cycle $W$.

\subsection{The Coating Thickness Superposition Cycle}

For constant two times spray and constant many times spray, $W=\delta$. For variable two times spray, the cycle $W$ is affected by the $k$, where $k$ is the ratio between the spacing distance of the second time spray path and the spacing distance of the first time spray path. Suppose the ratio is $1 / k(k>1)$, therefore the coating thickness superposition status cycle can be expressed as:

$$
W=\min P\{n / k\} \llbracket k \delta, n=1,2,3, \ldots, N
$$

Where $P$ is the set of integers.

\subsection{The Number of Coating Thickness Superposition Status}

The coating thickness superposition model is due to the different superposition status. Suppose the change direction of the offset distance between the first time spray painting trajectory and the subsequent spray painting trajectory along the positive $X$. First the coating thickness superposition status of two times spray painting is discussed, the coating thickness superposition model of two times spray painting change with the $x$-coordinate value of the boundary of the overlap region in process of the second time spray painting trajectory move from the origin $O$ to the $X$-positive. Such as in Figure 3, the gray area and the blank area are the overlap region and the non-overlap region. When status $1, x_{3}^{1}>x_{2}^{2}$, when status $2, x_{3}^{1}<x_{2}^{2}$. Therefore, taking into account the constant and the variable two times spray painting, the number $M$ of coating thickness superposition status can be expressed as:

$$
M=\frac{2 W}{\delta}+1
$$




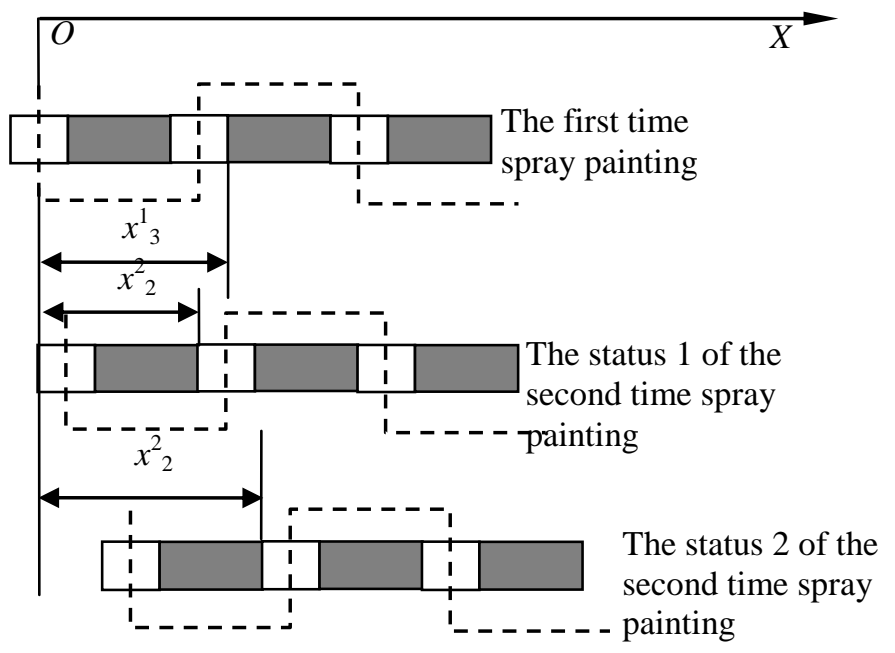

Figure 3. Diagram of Coating Thickness Superposition Status

There are three kinds of coating thickness superposition status for two times spray painting and each status is asymmetrical distribution in a cycle $W$. According to the changing principle of the coating thickness superposition status, after the third time spray painting, there are five superposition statuses in each superposition status of two times spray painting, so the number of coating thickness superposition status is fifteen when three times spray painting. Generally, when spray painting $J$ times, the number of coating thickness superposition status is:

$$
M=3 \prod_{j=3}^{J}\left(3+2^{j-2}\right)
$$

As you can see in the above formula, as the number of spray painting time increase, the coating thickness superposition status sharp increase and which brings the complexity of modeling and trajectory optimization.

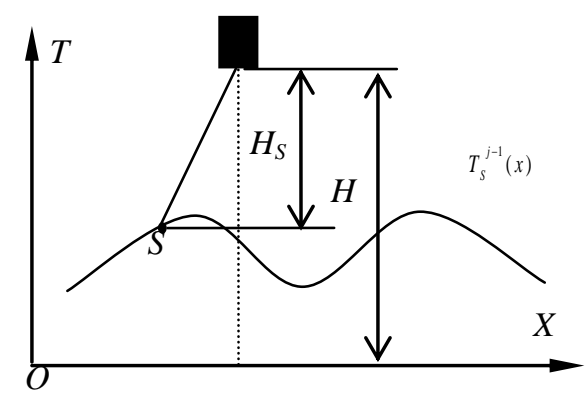

Figure 4. Actual Spray Height and Ideal Spray Height

\subsection{The Coating Thickness Superposition of Many Times Spray Painting}

When many-times spray painting, the coating thickness of each time spray painting can not simply superpose, because the shape of the workpiece surface is affected by the coating thickness of last spray painting, the surface shape has been displaced by the shape of coating thickness distribution. So when the next time spray painting, the coating thickness is grown 
based on the last coating thickness distribution, so the spray height is decreased than the last spray along the surface normal direction. Suppose the desired and the actual spray height are $H$ and $H_{S}$, the function of coating thickness distribution is $T_{s}^{j-1}(x)$ after the $j$-1 time spray painting, as shown in Figure 4, therefore there is:

$$
H_{S}=H-T_{S}^{j-1}(x)
$$

Suppose the coating thickness function of the $J$ time spray painting is $T^{j}(x)$ that relative to workpiece surface, the actual coating thickness function is $T_{s}^{j}(x)$, hence the coating thickness of any point $S$ on surface after $J$ times spray painting is:

$$
T_{S}^{j}(x)=T_{S}^{j-1}(x)+\eta^{j-1}(x) T^{j}(x)
$$

Where:

$$
\eta^{j-1}(x)=\left(\frac{H}{H-T_{S}^{j-1}(x)}\right)^{2}
$$

It represents the quotient of coating thickness based on the $J$-1 time spray painting.

\subsection{Spray Gun Trajectory Optimization of Two Times Spray Painting for Constant Parameters}

Suppose the ideal coating thickness of each time spray painting is equal when two times spray painting. Due to the spray gun parameters of each time spray are equal, so the optimized spacing distances $\delta$ of each time spray painting are equal. The offset distance $h$ between each spray gun trajectory needs to be optimized at this moment when two times spray painting, as shown in Figure 5 (a). In order to optimize the spray gun trajectory of two times spray painting, the coating thickness distribution must be modeling. Based on the cycle and status number of two times spray painting for constant parameters are $\delta$ and 3 , after two times spray painting, the coating thickness of any point $S$ on the surface is:

$$
\begin{aligned}
& \left\{\begin{array}{l}
T_{1}{ }^{1}+\eta^{1}(x) T_{1}{ }^{2} \quad m 1: h \leq x \leq \delta-R \\
\text { ins }
\end{array}\right. \\
& \left\{\begin{array}{l}
T_{1}{ }^{1}+T_{2}{ }^{1}+\eta^{1}(x) T_{1}{ }^{2} \\
T_{1}{ }^{1}+T_{2}{ }^{1}+\eta^{1}(x)\left(T_{1}{ }^{2}+T_{2}{ }^{2}\right) \quad m 1, m 2, m 3: h+\delta-R \leq x \leq R \\
m 2, m 3: h \leq x \leq h+\delta-R
\end{array}\right. \\
& T_{S}^{2}(x)=\left\{\begin{array}{l}
T_{2}^{1}+\eta^{1}(x)\left(T_{2}^{2}+T_{1}^{2}\right) \\
T_{3}: R \leq x \leq 2 \delta-R
\end{array}\right. \\
& \left\{\begin{array}{l}
T_{2}^{1}+\eta^{1}(x) T_{2}^{2} \\
m 1: R+h \leq x \leq \delta+h \\
m 2: R+h \leq x \leq 2 \delta-R
\end{array}\right. \\
& \left\{\begin{array}{l}
T_{2}{ }^{1}+T_{3}{ }^{1}+\eta^{1}(x) T_{2}{ }^{2} \\
m 3: R+h \leq x \leq \delta+h
\end{array}\right. \\
& \left(T_{2}{ }^{1}+T_{3}{ }^{1}+\eta^{1}(x)\left(T_{1}{ }^{2}+T_{2}{ }^{2}\right) \quad m 3: 2 \delta-R \leq x \leq R+h\right. \\
& m_{1}=\left\{0 \leq h_{1} \leq \delta-R\right\}, m_{2}=\left\{\delta-R \leq h_{2} \leq 2(\delta-R)\right\}, m_{3}=\left\{2(\delta-R) \leq h_{3} \leq \delta / 2\right\}
\end{aligned}
$$




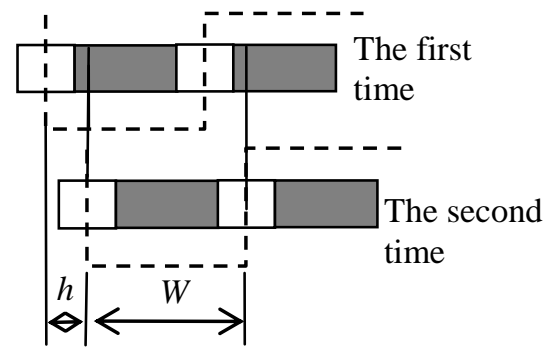

(a) constant

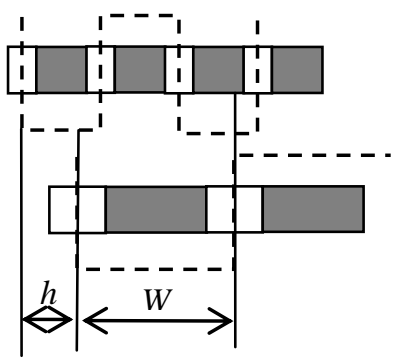

(b) variable

Figure 5. Constant and Variable Two Times Spray Painting

Where $T_{1}^{1}, T_{2}^{1}$ and $T_{3}^{1}$ are the ideal coating thickness of the first path, the second path and the third path on the first time spray painting trajectory, respectively; $T^{2}{ }_{1}$ and $T^{2}{ }_{2}$ are the ideal coating thickness of the first path and the second path on the second time spray painting trajectory, respectively; $m_{1}, m_{2}$ and $m_{3}$ are the value range of offset distance due to three different superposition statuses.

Due to three superposition statuses, the spray gun trajectory optimization of two times spray painting can be expressed as:

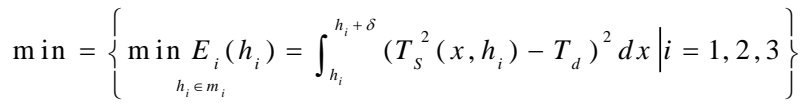

The meaning of the above formula is finds the $h$ when $E$ to minimum from tree different superposition status in range of thickness superposition cycle.

\subsection{Spray Gun Trajectory Optimization of Two Times Spray Painting for Variable Parameters}

A spraying way of variable parameters is used to reduce spray painting time in the actual spray painting production. Such as increase the spray radius and the flow to shorten the length of spray gun trajectory on a certain area of surface, on this account the spray painting time is reduced. Suppose the ideal coating thickness of each time spray painting is equal when two times spray painting, the spacing distance of optimized spray gun trajectory for the second time spray painting is $k \delta$ relative to the first time spray painting. Such as $k=2$, as shown in Figure 5 (b). The offset distance $h$ between each time spray gun trajectory needs to be optimized at this moment when two time spray painting. Based on the cycle $W$ and status number $M$ of two times spray painting for variable parameters are $2 \delta$ and 5 , after two times spray painting, the thickness of any point $S$ on the surface is: 


$$
\begin{aligned}
& \left\{\begin{array}{l}
T_{1}^{1}+\eta^{1}(x) T_{1}^{2} \\
m 2: h \leq x \leq \delta / 2
\end{array}\right. \\
& T_{1}^{1}+\eta^{1}(x)\left(T_{1}^{2}+T_{2}^{2}\right) \quad m 1: h+(\delta-R) / 2 \leq x \leq \delta / 2 \\
& T_{1}{ }^{1}+T_{2}{ }^{1}+\eta^{1}(x) T_{1}{ }^{2} \quad\{m 2: \delta-R \leq x \leq h+(\delta-R) / 2 \\
& \text { | }\left\{\begin{array}{l}
\text { m } 3, m 4, m 5: h \leq x \leq h+(\delta-R) / 2 \\
T_{1}+T_{2}+\eta^{\prime}(x) T_{1}
\end{array}\right.
\end{aligned}
$$

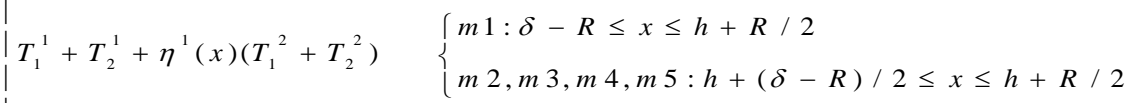

$$
\begin{aligned}
& T_{1}{ }^{1}+T_{2}{ }^{1}+\eta^{1}(x) T_{2}{ }^{2} \quad\left\{\begin{array}{l}
m 1, m 2, m 3: h+R / 2 \leq x \leq h+(2 \delta-R) / 2 \\
m 4, m 5: h+R / 2 \leq x \leq R
\end{array}\right.
\end{aligned}
$$

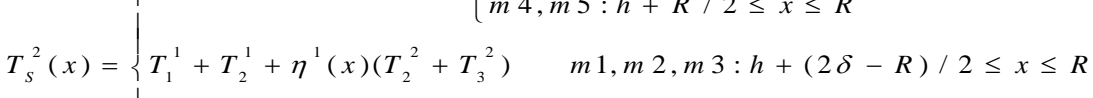

$$
\begin{aligned}
& \left\{\begin{array}{l}
T_{2}{ }^{1}+\eta^{1}(x)\left(T_{2}{ }^{2}+T_{3}{ }^{2}\right) \\
m 4: h+(2 \delta-R) / 2 \leq x \leq(\delta+R) / 2+h \\
m 5: h+(2 \delta-R) / 2 \leq x \leq 2 \delta-R
\end{array}\right. \\
& \left\{\begin{array}{l}
T_{2}^{1}+\eta^{1}(x) T_{3}^{2} \\
m 1, m 2:(\delta+R) / 2+h \leq x \leq \delta+h \\
m 3, m 4:(\delta+R) / 2+h \leq x \leq 2 \delta-R
\end{array}\right. \\
& T_{2}^{1}+\eta^{1}(x) T_{2}^{2} \quad m 4, m 5: R \leq x \leq h+(2 \delta-R) / 2 \\
& \left\{\begin{array}{l}
T_{2}{ }^{1}+T_{3}{ }^{1}+\eta^{1}(x) T_{3}{ }^{2} \\
m 5:(\delta+R) / 2+h \leq x \leq \delta+h
\end{array}\right. \\
& \left(T_{2}^{1}+T_{3}^{1}+\eta^{1}(x)\left(T_{2}^{2}+T_{3}^{2}\right) \quad m 5: 2 \delta-R \leq x \leq(\delta+R) / 2+h\right. \\
& m_{1}=\left\{0 \leq h_{1} \leq(\delta-R) / 2\right\}, m_{2}=\left\{(\delta-R) / 2 \leq h_{2} \leq \delta-R\right\}, m_{3}=\left\{\delta-R \leq h_{3} \leq(3 R-2 \delta) / 2\right\}, \\
& m_{4}=\left\{(3 R-2 \delta) / 2 \leq h_{4} \leq 1.5(\delta-R)\right\}, m_{5}=\left\{1.5(\delta-R) \leq h_{5} \leq R / 2\right\}
\end{aligned}
$$

Due to $M$ superposition statuses when $k>1$, the spray gun trajectory optimization of two times spray painting for variable parameters can be expressed as:

$$
\min =\left\{\min _{h_{i} \in m_{i}}\left(h_{i}\right)=\int_{h_{i}}^{h_{i}+W}\left(T_{S}^{2}\left(x, h_{i}\right)-T_{d}\right)^{2} d x \mid i=1,2, \cdots, M\right\}
$$

The meaning of the above formula is finds the $h$ when $E$ to minimum from $M$ superposition statuses in range of thickness superposition cycle $W$.

The solution of formula (6) and (7) belong to univariate optimization problem, the golden section method can be used to solve them under constrained condition.

\subsection{Spray Gun Trajectory Optimization of Many Times Spray Painting}

Generally speaking, the spray painting which the spray time more than two times is manytimes spray painting. Because the complexity of many-times spray painting for variable parameters, the many-times spray painting for constant parameters is developed in this paper, now the cycle $W$ is $\delta$. Suppose spray painting time is $J$, with regard to the trajectory optimization of many-times spray painting, need to optimize the offset distances that subsequent $J-1$ trajectories relative to the first trajectory, respectively, there are $J-1$ variables in here. Suppose the offset distance between the $j$ time spray painting trajectory and the first time spray painting trajectory is $h_{j}$, as shown in Figure 6 . For $J$ times spray painting, the spray gun trajectory optimization can be expressed as: 


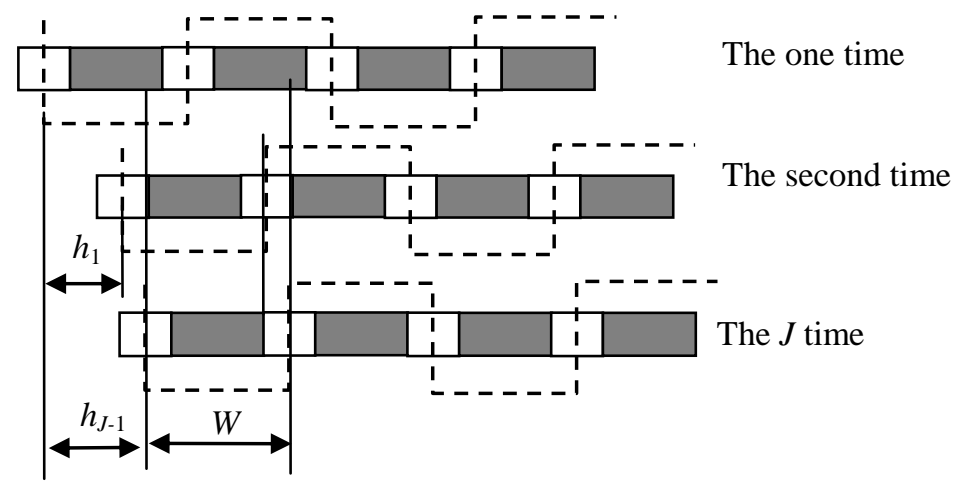

Figure 6. Constant Many Times Spray Painting

$$
\min =\left\{\begin{array}{l}
\min E_{i}\left(h_{1}^{i}, h_{2}^{i}, \cdots, h_{j-1}^{i}\right) \\
\begin{array}{c}
h_{1}^{i} \in\left\{m_{1}^{2}, m_{2}^{2}, m_{3}^{2}\right\} \\
h_{2}^{i} \in\left\{m_{1}^{3}, m_{2}^{2}, m_{3}^{3}, m_{4}^{3}, m_{5}^{3}\right\} \\
h_{j-1}^{i} \in\left\{m_{1}^{j}, m_{2}^{j}, \cdots, m^{j}, 3^{j} j^{j-2}\right\}
\end{array} \\
\left.=\int_{h_{j-1}^{i}}^{h_{j-1}^{i}+\delta}\left(T_{S}^{2}\left(x, h_{1}^{i}, h_{1}^{i}, \cdots, h_{j-1}^{i}\right)-T_{d}\right)^{2} d x \mid \begin{array}{c}
i=1,2, \cdots, M \\
j=3,4, \cdots, J
\end{array}\right\}
\end{array}\right\}
$$

Where $m_{3+2^{j-2}}^{j}$ is the value range of $h_{j-1}^{i}$ under the $\left(3+2^{j-2}\right)$ coating thickness superposition status when the $j$ time spray painting is performed; $h_{j-1}^{i}$ is the offset distance between the $j$ time spray gun trajectory and the first time spray gun trajectory under the $i$ coating superposition status. The meaning of the above formula is finds a group of value about $h$ when $E$ to minimum from $M$ superposition statuses in range of thickness superposition cycle.

Above formula belongs to multivariable optimization, for its solution can use the genetic algorithm under constrained condition [14], here no longer expatiatory.

\section{Simulation and Analysis}

Suppose the required average thickness $T_{d}$ is $50 \mu \mathrm{m}$, the spray radius $R=50 \mathrm{~mm}$ and the spray height $H=150 \mathrm{~mm}$. when vertical spray painting, from experimental data by fitting to obtain the paint deposition rate is:

$$
f(r)=0.1\left(R^{2}-r^{2}\right)
$$

In this paper, the operations of the optimization and numerical calculation are based on Matlab R2010a software.

\subsection{Simulation for One Time and Two Times Spray Painting}

In order to compare with the simulation results of one time spray painting in document [4], spray painting on a plane for one time. According to formula (1), the optimized spray velocity and the spacing distance are $323.2 \mathrm{~mm} / \mathrm{s}$ and $60.8 \mathrm{~mm}$, now the maximum and the minimum coating thicknesses are $52.0 \mu \mathrm{m}$ and $48.1 \mu \mathrm{m}$. Then spray painting on a plane for two times, suppose the required average thickness of each time is $25 \mu \mathrm{m}$, so the optimized spray velocity and the spacing distance of each time are $644.5 \mathrm{~mm} / \mathrm{s}$ and $60.8 \mathrm{~mm}$. According to formula (6), the optimized value $E$ of the optimization object function and the optimized value $h$ under three coating superposition statuses as follows: 
Table 1. Optimization Results for Constant Two Times Spray Painting

\begin{tabular}{ccc}
\hline$i$ & $E_{i}$ & $h_{i}(\mathrm{~mm})$ \\
\hline 1 & 24.4 & 10.8 \\
2 & 6.7 & 14.7 \\
3 & 19.9 & 21.6 \\
\hline
\end{tabular}

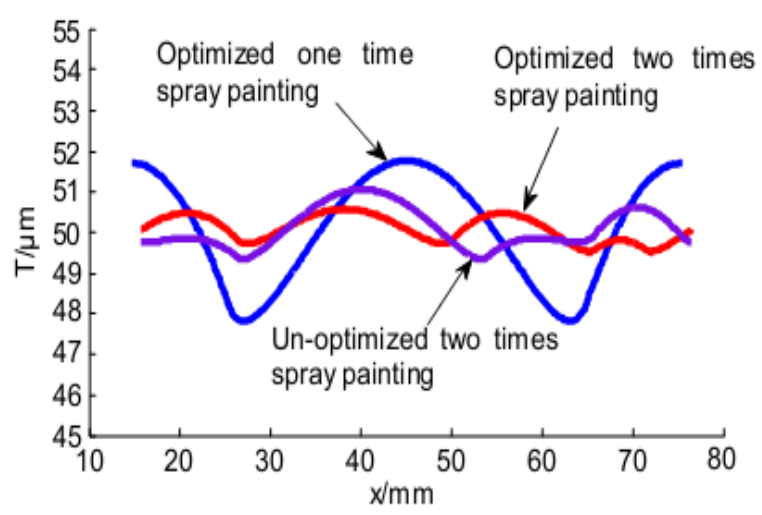

Figure 7. Thickness Distribution of one Time Spray painting, Optimized and noOptimized Constant Two Times Spray Painting

Table 1 shows the value $E_{2}$ achieve minimum and the corresponding value $h_{2}$ is $14.7 \mathrm{~mm}$, this moment, the maximum and the minimum coating thicknesses are $50.7 \mu \mathrm{m}$ and $49.7 \mu \mathrm{m}$. If the random value of the offset distance is $20 \mathrm{~mm}$, so the maximum and the minimum coating thicknesses are $51.1 \mu \mathrm{m}$ and $48.6 \mu \mathrm{m}$. Figure 7 shows the coating thickness distribution curves about one time spray painting of document [5] and two times spray painting of this paper in a single cycle.

Figure 7 shows that if take no account of optimization, take any value $h(h \neq n \delta$, $n=0,1, \ldots, N)$, the coating thickness uniformity of any two times spray painting is better than one time spray painting, and the coating thickness uniformity of optimized two times spray painting reach optimum.

\subsection{Simulation for Variable Two Times Spray Painting}

Suppose the required average thickness of each time is $25 \mu \mathrm{m}$, the spray radiuses of the first time and the second time spray painting are $25 \mathrm{~mm}$ and $50 \mathrm{~mm}$, respectively, that is $k=2$. The spacing distances of each time spray painting are $30.4 \mathrm{~mm}$ and $60.8 \mathrm{~mm}$ after optimized, respectively. According to formula (2) and (3), the number $M$ and the cycle $W$ of the coating thickness superposition status are 5 and $60.8 \mathrm{~mm}$, respectively. Once more, according to formula (7), the optimized value $E$ of the optimization object function and the optimized value $h$ under three coating thickness superposition statuses as follows:

Table 2. Optimization Results for Variable Two Times Spray Painting

\begin{tabular}{ccc}
\hline$i$ & $E_{i}$ & $h_{i}(\mathrm{~mm})$ \\
\hline 1 & 121.7 & 0 \\
2 & 140.1 & 5.4 \\
3 & 212.9 & 10.8 \\
4 & 243.1 & 14.2 \\
5 & 138.2 & 25.0 \\
\hline
\end{tabular}




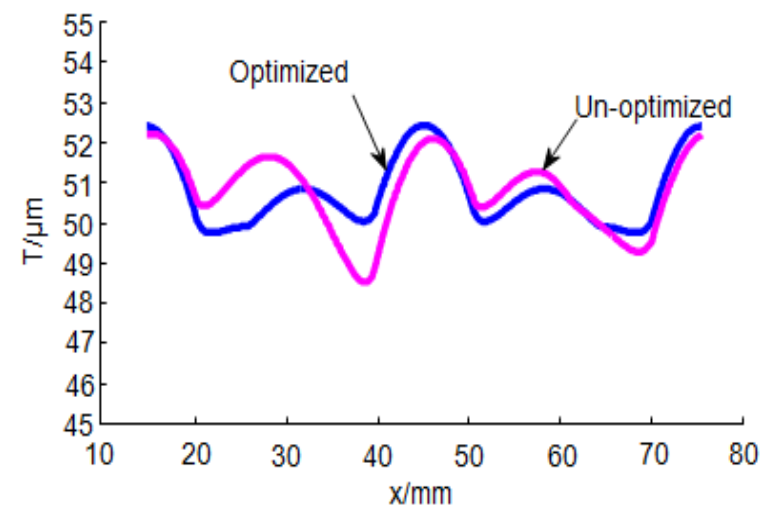

Figure 8. Thickness Distribution of the Optimized and Un-optimized Variable Two Times Spray Painting

Table 2 shows the value $E_{1}$ achieve minimum and the corresponding value $h_{1}$ is $0 \mathrm{~mm}$, this moment, the maximum and the minimum coating thicknesses are $52.4 \mu \mathrm{m}$ and $49.8 \mu \mathrm{m}$. If the random value of the offset distance is $25.4 \mathrm{~mm}$, so the maximum and the minimum coating thicknesses are $52.2 \mu \mathrm{m}$ and $48.6 \mu \mathrm{m}$. Figure 8 shows the coating thickness distribution curves of un-optimized and optimized variable two times spray painting.

From above figure know that the optimized thickness uniformity of variable two times spray painting is better than un-optimized. As you can see in the Figure 7 and Figure 8, the optimized thickness uniformity of constant two times spray painting is better than the optimized variable two times spray painting.

\subsection{Simulations of Constant Three Times Spray Painting}

Suppose spray time $J$ is 3 , the required average thickness of each time is $16.7 \mu \mathrm{m}$. The optimized spray velocity and the spacing distance of each time spray painting are $323.2 \mathrm{~mm} / \mathrm{s}$ and $60.8 \mathrm{~mm}$, respectively, the cycle $W$ is $60.8 \mathrm{~mm}$. According to formula (4), the number $M$ of coating thickness superposition status is 15 . Once more, according to formula (8), the optimized offset distances $h_{1}$ and $h_{2}$ of three times spray painting are $10.8 \mathrm{~mm}$ and $52.0 \mathrm{~mm}$, respectively, now the maximum and minimum coating thicknesses are $50.6 \mu \mathrm{m}$ and $49.7 \mu \mathrm{m}$. If the random values of the offset distance are $16.4 \mathrm{~mm}$ and $39.2 \mathrm{~mm}$, respectively, so the maximum and minimum coating thicknesses are $50.9 \mu \mathrm{m}$ and $49.2 \mu \mathrm{m}$. Table 3 shows the optimized value $E$ of constant two times and constant three times spray painting. Figure 9 shows the coating thickness distribution curves of optimized two and three times spray painting.

Table 3. Optimization Results Comparison between Constant Two Times and Constant Three Times Spray Painting

\begin{tabular}{cccc}
\hline & $E$ & $T_{\max }(\mu \mathrm{m})$ & $T_{\min }(\mu \mathrm{m})$ \\
\hline Three times & 1.7 & 50.6 & 49.7 \\
Two times & 6.7 & 50.7 & 49.7 \\
\hline
\end{tabular}




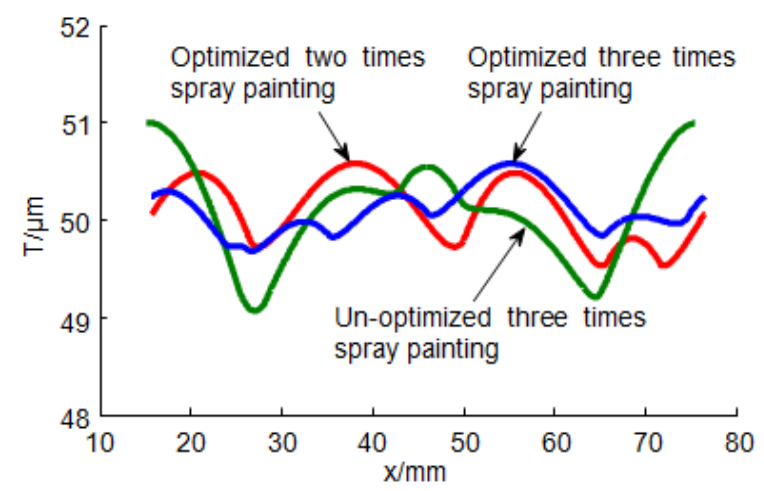

\section{Figure 9. Thickness Distribution of the Optimized Constant Two Times Spray Painting, the Optimized and Un- optimized Constant Three Times Spray Painting}

From the Table 3 and the Figure 9 know that the coating thickness uniformity of optimized three times spray painting is better than the optimized two times spray painting. In theory, the constant two times and three times spray painting are used to control thickness error in range of $\pm 1 \mu \mathrm{m}$, that meet strict requirements.

\section{Conclusions}

A new spray gun trajectory optimization method of many-times spray painting for the plane has been developed after complex surface partitioned. Based on the spray gun trajectory optimization of one time spray painting, the method utilizes the coating thickness superposition cycle and status number to establish the coating thickness superposition model for many-times spray painting and to optimize the spray gun trajectory of many-times spray painting. Simulation results showed that the paint thickness uniformity of arbitrary manytimes spray painting is promoted compare with one time spray painting. The optimized paint thickness uniformity of many-times spray painting has significantly improved. The optimized paint thickness uniformity of constant two times spray painting is better than the variable two times spray painting. The optimized paint thickness uniformity of three times spray painting is better than the optimized two times spray painting, but along with spray time increase, the spray gun trajectory optimization is becoming increasingly complexity, and the paint thickness uniformity does not necessarily continue to be promoted. Our future work will concentrate on some practical issues, such as the spray gun trajectory optimization at the junction of the patch and the patch because this will also affect coating thickness uniformity.

\section{Acknowledgements}

The project was supported by the National Natural Science Foundation of China (Grant No. 51165022), research fund of Key Laboratory for Advanced Technology in Environmental Protection of Jiangsu Province (Grant No. AE201322) and research fund of Jiangsu Key Laboratory of Large Engineering Equipment Detection and Control (Grant No. JSKLEDC201303).

\section{References}

[1] Y. H. Wang and N. Chi, "Path planning optimization for teaching and playback welding robot", Telkomnika, vol. 11, no. 2, (2013). 
[2] C. D. Conner, A. Greenfield, N. P. Atkar, A. A. Rizzi and H. Choset, "Paint deposition modeling for trajectory planning on automotive surfaces", IEEE Transactions on Automation Science and Engineering, vol. 2, no. 4, (2005).

[3] N. P. Atkar, A. Greenfield, C. D. Conner, H. Choset and A. A. Rizzi. "Hierarchical segmentation of surfaces embedded in \& Rdbl; ${ }^{3}$ for auto-body painting", Proceedings of the 2005 IEEE International Conference on Robotics and Automation, Barcelona, Spain, (2005) April 18-22.

[4] N. A. Prasad, G. Aaron, C. D. Conner, H. Choset and A. A Rizzi. "Uniform coverage of automotive surface patches", The International Journal of Robotics Research, vol. 24, no. 11, (2005).

[5] H. P. Chen and N. Xi, "Automated tool trajectory planning of industrial robots for painting composite surfaces", International Journal of Advanced Manufacturing Technology, vol. 35, no. 7-8, (2008).

[6] H. P. Chen, T. Fuhlbrigge and X. Z. Li, "A review of Cad-based robot path planning for spray painting", Industrial Robot, vol. 36, no. 4, (2009).

[7] W. H. Sheng, H. P. Chen and N. Xi, "Tool path planning for compound surfaces in spray forming processes", IEEE Transactions on Automation Science and Engineering, vol. 2, no. 3, (2005).

[8] D. A. Zhao, W. Chen and Y. Tang, "Tool path planning of spray painting robot based on genetic algorithms", China Mechanical Engineering, vol. 19, no. 7, (2008).

[9] W. Chen, D. A. Zhao and X. Y. Ping, "Tool path planning of spray painting robot based on ant colony algorithms", Machinery Design \& Manufacture, no. 7, (2011).

[10] W. Chen, D. A. Zhao and F. Z. Li, "Tool trajectory planning of robotic spray painting and its experiment for complex curved surfaces", Transactions of the Chinese Society for Agricultural Machinery, vol. 42, no. 1, (2011).

[11] F. Z. Li, D. A. Zhao and C. Zhang, "Trajectory optimization of spray painting robot based on CAD", Transactions of the Chinese Society for Agricultural Machinery, vol. 41, no. 5, (2010).

[12] W. Persoons and H. V. Brussl. "Cad-based robotic coating with highly curved surfaces", Proceedings of the 24th International Symposium on Industrial Robots, Tokyo, Japan, (1993) November 4-6.

[13] N. Asakawa and Y. Takeuchi, "Teachingless spray-painting of sculptured surface by an industrial robot", Proceedings of the 1997 IEEE International Conference on Robotics and Automation, Piscataway, USA, (1997) April 20-25.

[14] J. S. Jiang and C. X. He, "Editor, Optimization Calculation Method", South China University of Technology Publishers, Guangzhou, (2005).

\section{Authors}

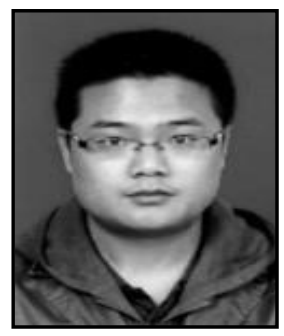

Yong Zeng, he was born in Jiangsu, China, in 1982. He received his Ph.D. degree in Mechanical Manufacture and Automation from Lanzhou University of Technology in 2011. His recent research interest is tool trajectory optimization of spray painting robot for complex surface.

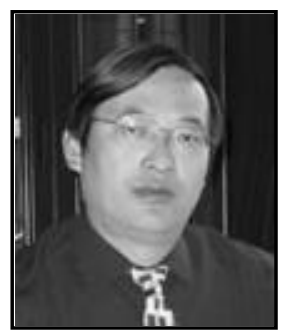

Jun Gong, he was born in Sichuan, China, in 1963. He received his M.S. degree in Mechanical Manufacture and Automation from Lanzhou University of Technology in 1992.

$\mathrm{He}$ is currently a professor and doctoral supervisor in school of Mechanical and Electronical engineering, Lanzhou University of Technology, China. His recent research interest is special equipment and its control system. 


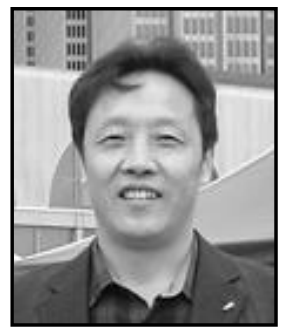

Ning Xu, he was born in Sichuan, China, in 1964. He received his Ph.D. degree in Mechanical Manufacture and Automation from Wuhan University of Technology in 2007.

$\mathrm{He}$ is currently a professor in Key Laboratory for Advanced Technology in Environmental Protection of Jiangsu Province, Yancheng Institute of Technology, China. His recent research interest is environmental protection equipment and its control system.

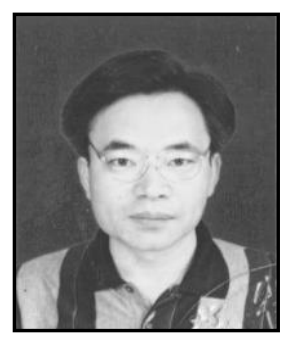

Nailing Wu, he was born in Jiangsu, China, in 1965. He received his master degree in Mechanical Manufacture and Automation from Shanghai University of Technology in 2005. His recent research interest is hydraulic control system. 
International Journal of Control and Automation Vol.7, No.8 (2014) 